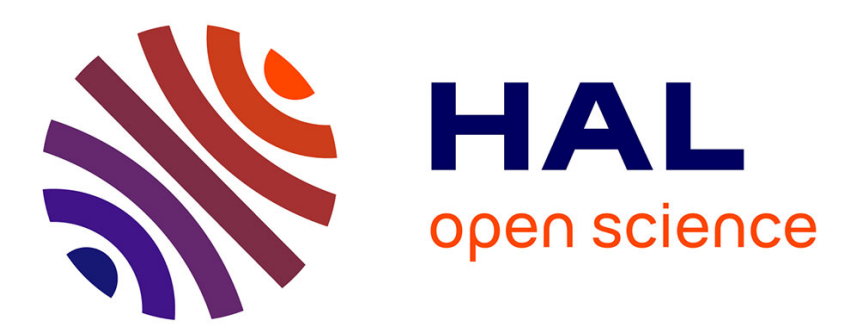

\title{
Assessing the planimetric accuracy of Paris atlases from the late 18th and 19th centuries
}

Bertrand Dumenieu, Nathalie Abadie, Julien Perret

\section{To cite this version:}

Bertrand Dumenieu, Nathalie Abadie, Julien Perret. Assessing the planimetric accuracy of Paris atlases from the late 18th and 19th centuries. Symposium on Applied Computing (SAC 2018), Apr 2018, Pau, France. pp.876-883, 10.1145/3167132.3167228 . hal-02388077

\section{HAL Id: hal-02388077 \\ https://hal.science/hal-02388077}

Submitted on 7 Dec 2019

HAL is a multi-disciplinary open access archive for the deposit and dissemination of scientific research documents, whether they are published or not. The documents may come from teaching and research institutions in France or abroad, or from public or private research centers.
L'archive ouverte pluridisciplinaire HAL, est destinée au dépôt et à la diffusion de documents scientifiques de niveau recherche, publiés ou non, émanant des établissements d'enseignement et de recherche français ou étrangers, des laboratoires publics ou privés. 


\section{Assessing the planimetric accuracy of Paris atlases from the late 18th and 19th centuries}

\author{
Bertrand Duménieu \\ CRH, EHESS \\ 54 boulevard Raspail \\ Paris, France 75006 \\ bertrand.dumenieu@ehess.fr
}

\author{
Nathalie Abadie \\ Univ. Paris-Est, LASTIG COGIT, IGN, \\ ENSG \\ 73 avenue de Paris \\ Saint Mandé, France 94160 \\ nathalie-f.abadie@ign.fr
}

\author{
Julien Perret \\ Univ. Paris-Est, LASTIG COGIT, IGN, \\ ENSG \\ 73 avenue de Paris \\ Saint Mandé, France 94160 \\ julien.perret@ign.fr
}

\begin{abstract}
The recent initiatives to digitize cultural heritage resources and publish them on the Web have renewed interest in historical maps for the diachronic analysis of territories in GIS applications. However, such analyses should not be done without a good understanding of the possibilities and limitations of geographical information provided by historical maps, i.e. their quality. One of the major concerns regarding historical maps quality is their positional planimetric accuracy which highly depends on survey techniques used at the time. As these techniques are not always thoroughly known and as ground truth is most of the time not sufficiently available, direct absolute evaluation approaches have been proposed to assess historical maps positional planimetric accuracy. In this article, we follow the intuition that the most widely adopted georeferencingbased approach for assessing the positional planimetric accuracy of historical maps can be adapted to provide an evaluation of the error caused by the survey process in cases like Paris atlases where the georeferencing transformation can be estimated with ground control points based on geodetic features and where the projection of the map can be approximated by a well known projected coordinate reference system. We apply this tuned approach on the Verniquet atlas and evaluate the validity of our hypothesis about projection approximation.
\end{abstract}

\section{CCS CONCEPTS}

-Information systems $\rightarrow$ Geographic information systems; Digital libraries and archives; Information retrieval;

\section{KEYWORDS}

planimetric accuracy assessment; historical maps

\section{ACM Reference format:}

Bertrand Duménieu, Nathalie Abadie, and Julien Perret. 2018. Assessing the planimetric accuracy of Paris atlases from the late 18th and 19th centuries. In Proceedings of SAC 2018: Symposium on Applied Computing, Pau, France, April 9-13, 2018 (SAC 2018), 8 pages.

DOI: $10.1145 / 3167132.3167228$

SAC 2018, Pau, France

(C) 2018 Copyright held by the owner/author(s). Publication rights licensed to ACM. This is the author's version of the work. It is posted here for your personal use. Not for redistribution. The definitive Version of Record was published in Proceedings of SAC 2018: Symposium on Applied Computing , April 9-13, 2018, http://dx.doi.org/10. 1145/3167132.3167228

\section{INTRODUCTION}

Comparing the content of maps drawn at different points in time to bring out changes in a geographical region is a commonly used method for the diachronic analysis of territories[4, 17]. In this context, historical maps are crucial sources of historical evidence bearing testimony to spatial configurations that have partially or entirely changed. Beyond picturing a territory, historical maps give away the state of mapping technologies and scientific knowledge of their time. They form raw material for the history of science, the study of long-term territorial dynamics and more generally for the analysis of any historical phenomenon whose understanding might benefit from a space-centered approach.

The widening diffusion of GIS within social sciences, concomitant with digital preservation policies in libraries and museums, have raised interest in historical maps as data sources of direct spatial references, i.e drawings of geographical entities with their shape and location. As a result, initiatives such as [12] or [16] have taken advantages of historical maps to produce geo-historical datasets by locating each map in a coordinate reference system and extracting the geographical entities set in them.

When exploiting geographical data, assessing its positional accuracy and spatial uncertainty is of paramount importance because these two elements could greatly affect the results of spatial analyses.This is even more true for data extracted from historical maps because in situ verifications are excluded and because such data is subject to a higher degree of inaccuracy and uncertainty than modern data.The absolute positional planimetric accuracy of geographic data is defined by the ISO 19157 standard on geographic data quality [6] as the "closeness of reported coordinate values to values accepted as or being true".

Spatial uncertainty is related to the quantity of information lost through the chain of cognitive and technical sub-processes which form at a higher level the map making and map reading processes $[13,15]$. The concept of topographic accuracy/error (Blackemore, Laxton) is very close.For the sake of readability, we use accuracy to refer to both concepts equally.

Many approaches have been proposed to evaluate the positional accuracy of geographic data. The ISO 19157 standard distinguishes between direct and indirect methods of quality evaluation. Indirect methods are based on knowledge about data, often provided by genealogical information. However, in the case of historical maps, such information is most of the time unknown or incomplete. Direct methods rely on the inspection of the data, either by analyzing them on their own (absolute methods) or by comparing them with other data sources (relative methods). The catalog of standardized 
quality measures of the ISO 19157 standard proposes many numerical methods to evaluate the positional planimetric accuracy of geographic data. Unfortunately, relative methods require a high quality reference data source, which is most of the time lacking when dealing with geo-historical data.

In this article, we focus on a particular subset of historical maps: the atlases of Paris drawn during the late $18^{\text {th }}$ century and the $19^{\text {th }}$ century. These large scale, high dimension maps have several properties which can be used to finely assess their positional accuracy. The remainder of this article is organized as follows: Section 2 presents related works in the field of historical maps positional planimetric accuracy evaluation and introduces some characteristics of Paris atlases that may be used to overcome the limitations of state of the art positional accuracy assessment approaches. In section 3 we propose to extend the georeferencing approach most commonly used for old maps in order to produce an in-depth analysis of the planimetric accuracy of Paris atlases. In Section 4, we apply this approach on the Verniquet atlas and we evaluate the validity of our hypothesis.

\section{STUDYING THE GEOMETRIC PROPERTIES OF HISTORICAL MAPS: THE CASE OF THE PARISIAN ATLASES}

In this section, we explain how georeferencing methods are used to assess the positional planimetric accuracy of ancient maps. As stated in many related works, assessing the quality of ancient maps require an in depth analyses of their genealogy in order to take their technical characteristics into account. We thus present some technical properties of interest of the Parisian atlases from the late 18th and 19th centuries, with a focus on the Edmé Verniquet atlas.

\subsection{Assessing the positional accuracy of ancient maps}

Years before the GIS era, historians of cartography used to overlap modern and old maps to study the positional accuracy of the latter. Although simple on the outside, this operation requires detailed knowledge of the genesis of the map and of the technical, scientific and political context surrounding the production of the map. As an example, Laxton [9] analyzed the positional accuracy of more than a hundred maps of English and Welsh county maps surveyed between 1740 and 1840 . This study rely on a mutual communication between a historical research and a visual comparison between these maps and a modern Ordnance Survey map of UK. It is noteworthy that Laxton distinguishes two categories of error affecting the global accuracy of a map: geodetic errors resulting from imperfections in the astronomical measurements prior to the survey, and planimetric errors resulting from the topographic surveying. The democratization of graphical GIS enabled historians to measure the positional accuracy of old maps with georeferencing tools.

Georeferencing refers to a set of image registration methods were each pixel of an image depicting a geographical region (e.g. a map) is assigned coordinates on the Earth surface. All these methods aim for the same goal: to find an interpolation between a non-georeferenced source image and a reference image portraying the same region with known geographical coordinates. This interpolation is used to warp the source image so the representation of the same geographical entity in both images are superimposed; once done, finding the geographical coordinates of the warped source image becomes straightforward.

In the case of maps, finding a satisfactory interpolation function is a two step process. First, a set of ground control points (GCPs) is selected, corresponding to geographical entities found in both the historical map and a previously chosen reference map. These GPCs are then used to compute the $2 \mathrm{D}$ interpolation between the source and the reference maps. Depending on the class of interpolation function, this can be done with optimization methods (approximate or exact like Thin Plate Splines) [11] or statistical regression [10]. With historical maps, it is frequently advised not to rely on exact methods since they lead to perfect matches and thus tend to artificially fade the uncertainties inherent to historical maps.

The $2 \mathrm{D}$ residuals produced by regression models are commonly used to measure the quality of historical maps. Indeed, residuals on each GCP can be read as the sum of all errors affecting the map in the vicinity of the GCP, and the direction of the residual vectors indicates how the map is locally distorted compared to the reference map. Residuals of regression models are an approximation of the planimetric accuracy of the historical map if the planimetric error of the reference map and the GCPs are negligible. They are also a measure of the distortion between the historical map and the reference map. Measures of regression quality like root mean squared error (RMSE) is then an global indicator of the planimetric accuracy of the old map and allow for quantitative and qualitative analyses of this error [2,3]. If the projection of the old map is well known, using linear interpolation models an makes possible to detect systematic errors in the geodetic properties of the old map such as a bad alignment with the prime meridian of the projection [2].

All these possibilities have been put together in the MapAnalyst software [7] which provide several visualization tools dedicated to the qualitative study of the positional accuracy of historical maps. Unlike georefencing tools, MapAnalyst does not warp the old map but instead computes the inverse transformation. Doing this allow to visualize the accuracy of the old map in its own the system, without having to warp it. This software has been rapidly adopted by the community and is today a standard tool in most of the recent papers on the geometrical analysis of old maps $[1,8,17]$.

\subsection{Properties of the 18-19th c. Parisian atlases}

The fast progresses of astronomy and scientific instrumentation in Europe during the early modern period caused a "race for accuracy" which resulted in the creation of many topographic maps (e.g. the Cassini map of France). On the other side of the map scale, urban maps followed the same path: at least in western Europe, isometric and bird-eye views were gradually abandoned during the $18^{\text {th }}$ century in favor of orthogonal views based on triangulated canvas. Located at the intersection point of the Paris meridian and a "main" parallel well known at this time (going through the Royal Observatory), Paris is a perfect candidate for high-precision cartography. Several times during $18^{\text {th }}$ and $19^{\text {th }}$ centuries will Paris be triangulated and mapped by surveyors, architects and geographers. From the beginning, these maps: 
- aim the highest level of planimetric accuracy to show the "true" face of the city to help the city and royal administrations in the management of the street network.

- use a Plate-Carrée projection where the Paris meridian is used as the central meridian and the automecoic parallel intersects the central meridian at the Royal Observatory of Paris.

The latter property allows for fine-grained analysis of the planimetric accuracy of the map content.

\subsection{The Atlas of Paris by Edmé Verniquet, 1785-1791}

In 1783 the French government enacted a series of laws introducing a proto-version of the règle de prospect by constraining the width of new streets and linking the maximum height of adjacent buildings to this width. Two years later, the architect Edmé Verniquet started to map the houses and streets of Paris. This immense amount of work ended in 1791 in the publication of a 1:1728 map of Paris divided into 72 sheets which has been used by the Paris administration as a reference topographic map of the city at least until the 1820 's (see Figure 1). The final map portrays the streets, city blocks along, main buildings and gardens on an area covering Paris and its suburbs at the time. Overlapping the geographical content, a $100 \times 100$ toises measured grid $(1$ toise $\approx 1.95 \mathrm{~m}$ ) is also drawn on the map. As in other Parisian maps, this grid is aligned with the geodetic lines of the Plate-Carrée projection mentioned before, i.e. the central meridian (resp. the automecoic parallel) overlaps one vertical (resp. horizontal) line of the grid.

\section{A GEOREFERENCING BASED APPROACH}

As stated in Section 2.1, georeferencing-based methods for the evaluation of the planimetric accuracy of historical maps take advantage of the $2 \mathrm{D}$ regression residuals.

The general idea is that the RMSE of these residuals reveals the distortion of the historical map compared to a reference map. If we consider the planimetric error of the reference map to be negligible when compared to the distortion of the historical map, then we measure the planimetric accuracy of the historical map. Two main sources of error explain the measured distortion:

- Intrinsic error of the historical map, due to errors in the surveying process (a) and distortions caused by the physical deterioration of the map (b). The evaluation of this intrinsic error is the goal of most studies on historical maps accuracy.

- Extrinsic error resulting from the georeferencing process. First, the location of every GCP is tainted by uncertainty caused by the inherent lack of precision in manual positioning (c), worsen by the gap between the mapping choices in the source and reference map (e.g. map generalization, scale). Secondly, the geodetic properties of the projection of an historical map is often poorly known (d). Possible shifting between the projection of the source and reference map will thus be embedded in the resulting distortion.

In studies on small scale maps such as country maps, error (d) is often considered as insignificant compared to (a) and (b) [7].
Strangely, error (c) is hardly taken into account if not in the general rule of "placing the GCPs carefully".

With large scale maps such as the Paris atlases, the geodetic error (d) cannot be neglected anymore since it may strongly affect the final distortion measurement. Some specificities of the $18-19^{\text {th }}$ centuries Parisian atlases come here in handy. Being relatively recent and engraved on high quality paper, the paper distortion is expected to be low. The large scale of the maps (e.g. 1:1728 for the Verniquet atlas) along with the low level of cartographic generalization decrease the risk of large GCP-positioning errors. Most of all, these maps portray two very different types of entities, i.e. geographic and geodetic.

We propose here an approach which, in the case of the Parisian atlases and under the hypothesis of low GCP positioning errors and paper distortion, exploits the twofold nature of their content to separate the geodetic and surveying errors. Exploring the survey errors enables a fine-grained critical analysis of the content of these atlases.

\subsection{Methodology overview}

The main goal of our methodology is to separate the survey error from other sources of error in residuals. We propose to take advantage of the measured grid to georeference the maps and estimate the parameters of a geometric interpolation that corrects all types of errors but the survey error. Indeed, the grid is related to geodetic entities such as the Paris meridian which are not affected by survey errors. Moreover, we can reasonably consider the planimetric accuracy of the grid to be very high, which allow us to measure the planimetric accuracy of Verniquet atlas. To do so, we use GCPs defined on the measured grid: this requires to define this grid in both the historical map and a target coordinate reference system.

Despite the grid being already depicted on the atlas, it must be reconstructed in the target coordinate reference system. In order to avoid geodetic errors due to discrepancy in the historical and reference map projections, we propose to approximate the Plate-Carrée used in Parisian atlases with a modern days projected coordinate reference system, namely Lambert I. This is possible because both the maps projection and Lambert I use the Paris meridian as central meridian and because the linear drift of Lambert I around Paris is very small (approx. $6 \mathrm{~cm} / \mathrm{km}$ ). Then, nodes of both grids are matched to serve as GCPs in the estimation of the georeferencing transformation.

In a second phase, we define a set of measure points captured on geographical entities depicted on the historical map and on a reference map projected in Lambert I. Applying the geometric transformation computed with the grid GCPs, we warp each measure point in the historical map to obtain its predicted location in Lambert I. The vector between this location and the location of the corresponding point in the reference map reflects the survey error at the location of this point in the historical map.

\subsection{Placing the grid GCPs}

Ground control points have known location in the image coordinate system of the historical map and in the coordinate reference system of the target map. We define GCPs to be placed. 


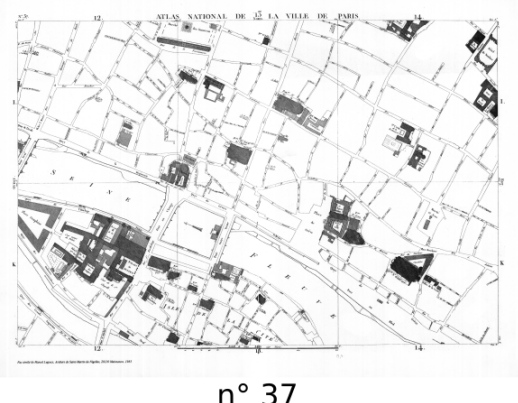

$n^{\circ} 37$

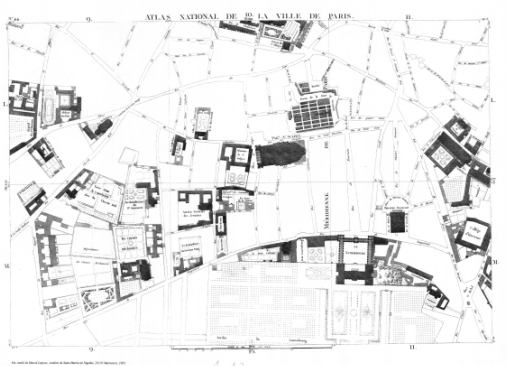

$n^{\circ} 44$

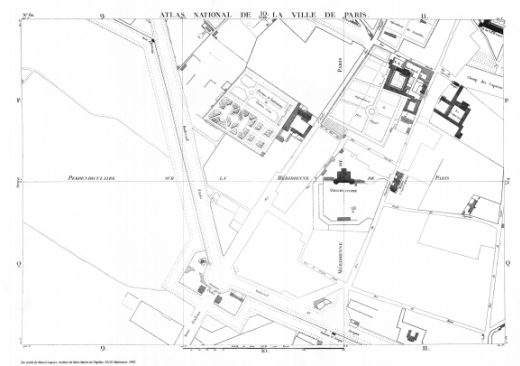

$n^{\circ} 60$

Figure 1: Three sheets of the Atlas of Paris by Edmé Verniquet.

Since the measured grid of the maps are built upon the geodetic lines of the map projection, a reasonable assumption is to those grids as graticules.

\subsection{Choosing the geometric transformation}

As stated above, the historical maps that we want to georeference and analyze are assumed to have the following properties:

- tiny paper distortion,

- GCPs based on geodetic features with no survey errors and no manual positioning errors,

- the same projected coordinated reference system as the target map.

In such conditions, the estimated geometric transformation should have a zero RMSE value. This is possible only with non-rigid transformation models: that is why this kind of geometric transformation functions should be used preferably. In cases where global transformation models are the only geometric transformation functions easily available, the function that produces the lower RMSE value should be used preferably.

\subsection{Choosing the measure points and assessing the positional accuracy of map content}

Measure points are points located on geographic features represented in the historical map and in some reference map, chosen for its high and well documented quality. Their position measured in the historical map is transformed with the estimated geometric function to produce an estimated position in the reference map. The vector defined by the location of the corresponding measure point from the reference map with its estimated position is then considered as the local survey error of the historical map.

The positional accuracy of the reference map should thus be higher as possible than the positional accuracy of the analyzed historical map and, even more importantly, known in details. Moreover, the reference map should also be chosen to be as close to the old map as possible in terms of level of detail, generalization and legend in order to minimize errors when defining the measure points.

The most widely accepted rule of thumb for the best efficiency of measure points suggests [5] to find a balance between (1) the regularity of their spatial distribution and (2) their number.

\section{ASSESSING THE POSITIONAL ACCURACY OF THE VERNIQUET ATLAS}

We apply the presented approach on the Verniquet atlas.

\subsection{Georeferencing the Verniquet atlas}

The first step is to build a grid with Lambert I coordinates corresponding to the measured grid of the atlas. To do so the position of the point of intersection of the Paris meridian and its parallel passing through the Paris observatory must be known in Lambert I. If the easting of this point is immediate (since the Paris meridian is the central meridian of Lambert I), finding its northing is less straightforward. However, we can find this point drawn on an orthogonal view of the Observatory drawn by Verniquet as a preliminary work [14](in French). Since we consider the grid to be approximately a graticule, we simply create a $195 \mathrm{~m} \times 195 \mathrm{~m}$ grid with Lambert I coordinates, where one of its node coincide with the point of intersection computed before.

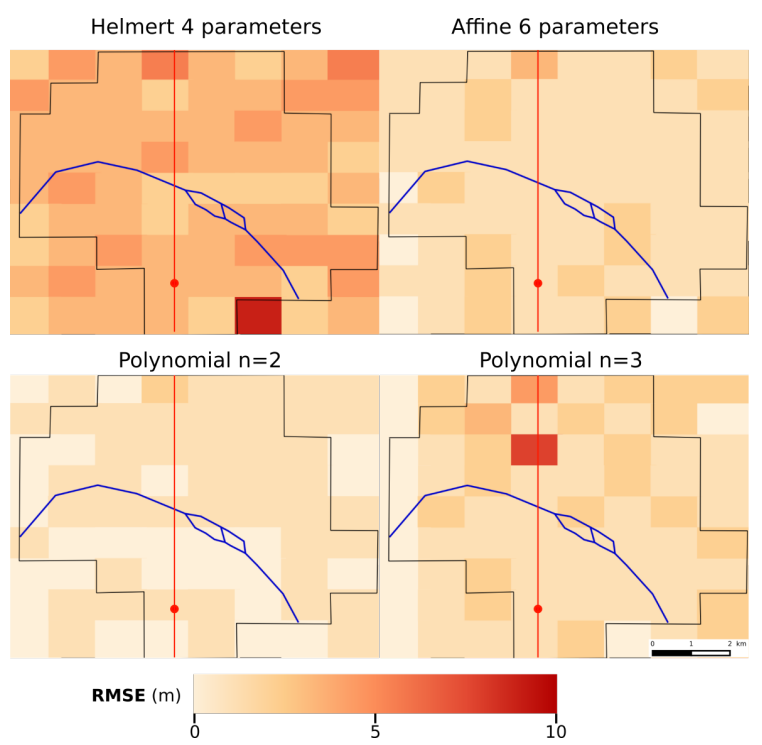

Figure 2: RMSE of each sheet of the Verniquet atlas using different geometric transformation models. 
Then, each sheet of the atlas is georeferenced using the nodes of the measures grid as GCPs. In order to reach the georeferencing with the minimum RMSE, we have tested several geometric transformation models: Helmert with 4 parameters, affine with 6 parameters and polynomial transformations (order 2 an 3).

Figure 2 illustrates the RMSE of each sheet with different transformations. To produce these maps, we georeferenced each sheet of the atlas on the reference grid (9 GCPs per sheet) and computed the RMSE on each set of residuals. This gives us the global distribution RMSE per sheet, i.e. the deviation between each georeferenced sheet and the reference grid. The model with the minimum mean $m$ and standard deviation $\sigma$ is preferred. The best model was a second order polynomial transformation $(\mu=1.1 \mathrm{~m}, \sigma=0.3 \mathrm{~m})$, which has been consequently used as the geometric transformation to measure the survey errors of the atlas.

\subsection{Analyzing the survey errors for two cartographic themes: the street network and the public buildings}

Two sets of measure points have been defined. First, a set of 779 measure points have been placed on the corners of the streets portrayed in the Verniquet atlas as well as in the modern parcel map of Paris, here used as the reference map in Lambert I. Another set of 523 measure points have been defined on the public buildings scattered in the map, using the same reference map. We applied the polynomial transformation computed before on those two sets of points. Figure 3 illustrates the obtained error vectors which carry the survey error.

\subsection{Evaluation of the georeferencing based approach}

One of the main assumptions of our approach is (a) to approximate the historical atlas projection with Lambert I and (b) using the grid traced on the map as a graticule. In this section, we propose to verify this hypothesis with the help of the measure points. A wrong hypothesis would result in a drift between the graticule in Lambert I and the grid in the atlas. Such a drift would lead to a systematic error visible in the distortion vectors measured by the points on streets and buildings. In order to detect this potential error and thus test our initial hypothesis, we rely on both a qualitative visual analysis and a quantitative analysis of the distortion vectors.

First, we map the $\mathrm{x}$-axis and $\mathrm{y}$-axis components of the distortion vector to show the spatial distribution of distortion on each dimension. To be able to detect the presence of a systematic error on each component, we use the Moran's Indice, which measures the spatial autocorrelation of a set of spatially located measures within a given distance. The Morans's Indice is close to 1 (resp. -1) in case of positive autocorrelation (resp. negative) and close to 0 when there is no correlation between measurements. In order to look for systematic errors at different scales, we compute the Moran's Indice with distances going up to $7600 \mathrm{~m}$ (maximum distance between measure points). The final results are displayed using correlograms.

4.3.1 Horizontal and vertical systematic errors. In order to search for a systematic error, we explore the potential vertical and horizontal drifs of the grid. A horizontal drift would reveal an error in the location of the central meridian, meaning that the Paris meridian is not located at the same place in the atlas and in the Lambert I projection.

Mapping the horizontal component of the distortion vectors for streets and buildings results in Figure 4, which shows no obvious systematic errors for both point sets. The correlogram of these horizontal distortions (see Figure 5) shows only very local correlations that are statistically significant (red points) for both cartographic themes, which is easily explained by the fact that close points tends to be affected by similar survey error.

We do the same for the vertical distortions (see Figures 6 and 7). Again, there is no clear evidence of a systematic error apart from the same local positive autocorrelation as for the horizontal distortion. This first evaluation tends to validate the fact that Lambert I is a reasonable approximation of the projection used in the Verniquet atlas and thus in all Parisian atlases of the $19^{\text {th }}$ century built on the same principles.

4.3.2 Remarks on the streets and buildings in the Verniquet atlas. A quick look at the maps of distortion vectors for both cartographic themes show very different figures of the survey errors in the map, yet these errors are always very small: 0 to 3.2 meters for the buildings, 0 to 2.5 meters for the street corners. Such distortion values are remarkable for a map created almost 230 years ago, placing the georeferenced Verniquet atlas as a candidate reference map for the georeferencing of old maps of Paris, especially for early modern era. The same figures shows that streets and buildings distortion seems to be very different. Although the low amplitude of these distortion order only a cautious conclusion, this might indicate that these two cartographic themes were not surveyed at the same time. This hypothesis is historically confirmed by [14], the buildings and streets originating from two completely different survey operations.

\section{CONCLUSION AND FUTURE WORKS}

In this article we explain how the georeferencing-based approach used for assessing the positional planimetric accuracy of historical maps can be adapted to provide a fine-grained evaluation of the errors due to the survey process in the case of maps having specific properties such as Paris atlases from the late 18th and the early 19th centuries. Our approach is based on the estimation of a georeferencing transformation function assuming that the historical and reference maps are defined in the same projected coordinate reference system and that GCPs are based on geodetic features and therefore free from survey errors. The application of this estimated transformation function on measure points picked on geographic features existing in both the historical and reference maps brings their associated survey errors out. We thus applied this approach on the Verniquet atlas, chosen as a typical example of Paris urban maps from the late 18th and the early 19th centuries. We analyzed the survey errors affecting the street network and the public buildings in this map. Finally, we evaluated the validity of our base hypothesis by analyzing the distortions caused by the estimated transformation on the main geodetic features of the Verniquet atlas.

In this work, we have implemented our approach by estimating the parameters of a second order polynomial transformation function. This kind of transformation function has the advantage of 


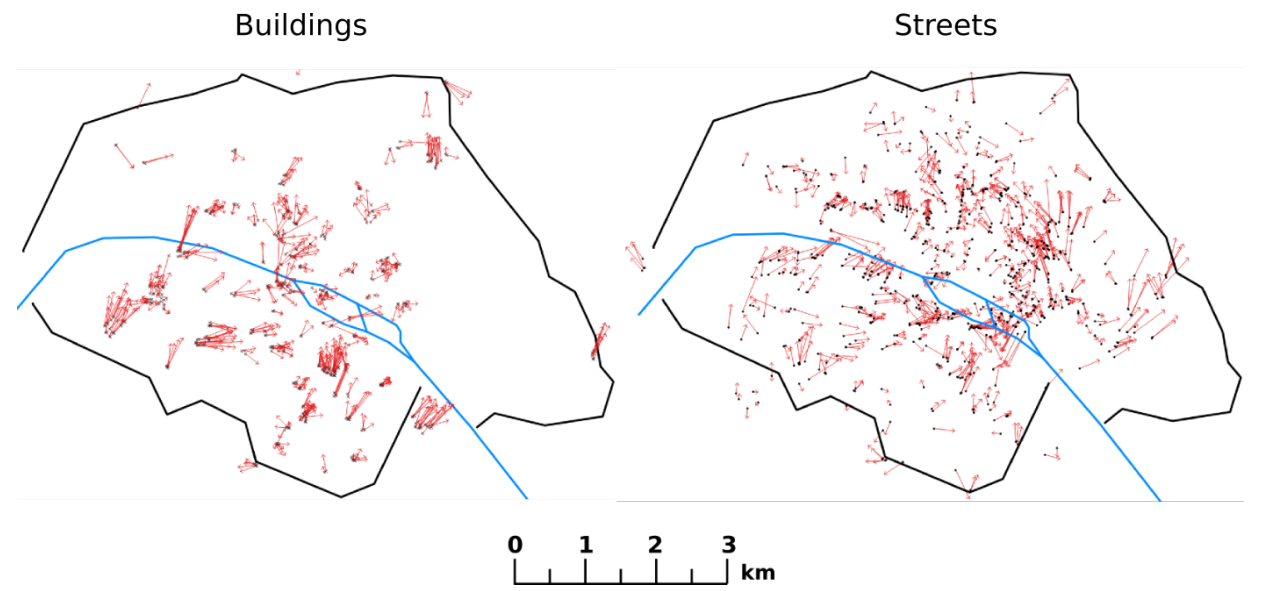

Figure 3: Error vectors measured on the street corners and buildings (exaggerated 100 times)

being commonly available in state-of-the art GIS libraries. But, as a global transformation function, it also has the drawback of having a non-zero RMSE value. Using a non-rigid transformation model like the thin plate spline function would provide an estimated transformation function completely free from residuals. Moreover, a tight evaluation of our assumptions regarding paper distortion or manual positioning of GCPs should also be performed in future work.

As survey errors are more likely to affect small-scale maps and as the they may be significantly higher is such cases than any other types of errors, our approach seems more suited for the analysis of the quality of small-scale maps whose geodetic canvas is well known, such as the Cassini map.

\section{ACKNOWLEDGMENTS}

The authors would like to thank the geodesy team of the French national mapping agency (IGN) for their insightful reviews and in particular Thierry Gattacceca and Gilles Canaud. The authors are also grateful to the anonymous reviewers for their helpful suggestions.

\section{REFERENCES}

[1] Andrzej Affek. 2013. Georeferencing of historical maps using GIS, as exemplified by the Austrian Military Surveys of Galicia. Geographia Polonica 86, 4 (2013), 375-390.

[2] Caterina Balletti. 2006. Georeference in the analysis of the geometric content of early maps. e-Perimetron 1, 1 (2006), 32-42.

[3] Maria A Brovelli and Marco Minghini. 2012. Georeferencing old maps: a polynomial-based approach for Como historical cadastres. e-Perimetron 7, 3 (2012), 97-110.

[4] Branka Cuca, Raffaella Brumana, Marco Scaioni, and Daniela Oreni. 2011. Spatial data management of temporal map series for cultural and environmental heritage. IFSDIR 6 (2011), 97-125.

[5] Pierre Alexis Herrault, David Sheeren, Mathieu Fauvel, Claude Monteil, and Martin Paegelow. 2013. A comparative study of geometric transformation models for the historical" map of france" registration. Geographia Technica 1 (2013), $\mathrm{pp}-34$.

[6] ISO. 2013. 19157: Geographic information - Data quality. International Standard. International Organization for Standardization (http://www.iso.org)

[7] Bernhard Jenny and Lorenz Hurni. 2011. Studying cartographic heritage: Analysis and visualization of geometric distortions. Computers \& Graphics 35, 2 (2011) 402-411.

[8] Iason Jongepier, Tim Soens, Stijn Temmerman, and Tine Missiaen. 2016. Assessing the planimetric accuracy of historical maps (sixteenth to nineteenth centuries): new methods and potential for coastal landscape reconstruction. The Cartographic fournal 53, 2 (2016), 114-132.

[9] Paul Laxton. 1976. The Geodetic and topographical evaluation of English county maps, 1740-1840. The Cartographic fournal 13, 1 (1976), 37-54.

[10] Evangelos Livieratos. 2006. On the study of the geometric properties of historical cartographic representations. Cartographica: The International fournal for Geographic Information and Geovisualization 41, 2 (2006), 165-176.

[11] Francisco Manzano-Agugliaro, Carlos San-Antonio-Gómez, Sergio López, Francisco G Montoya, and Consolación Gil. 2013. Pareto-based evolutionary algorithms for the calculation of transformation parameters and accuracy assessment of historical maps. Computers \& geosciences 57 (2013), 124-132.

[12] Julien Perret, Maurizio Gribaudi, and Marc Barthelemy. 2015. Roads and cities of 18th century France. Scientific data 2 (2015).

[13] Brandon Plewe. 2002. The nature of uncertainty in historical geographic information. Transactions in GIS 6, 4 (2002), 431-456.

[14] Jeanne Pronteau. 1986. Edmé Verniquet, architecte et auteur du "grand plan de Paris"(1785-1791). Paris, Commission des travaux historiques de la Ville de Paris (1986), 485.

[15] Konstatin A SALICHTCHEV. 1978. Cartographic communication/its place in the theory of science. Cartographica: The International fournal for Geographic Information and Geovisualization 15, 2 (1978), 93-99.

[16] Humphrey Southall, Paula Aucott, Chris Fleet, Tom Pert, and Michael Stoner. 2017. GB1900: engaging the public in very large scale gazetteer construction from the Ordnance Survey "County Series" 1: 10,560 mapping of Great Britain. fournal of Map \& Geography Libraries 13, 1 (2017), 7-28.

[17] Michele Tucci, Alberto Giordano, and Rocco W Ronza. 2010. Using spatial analysis and geovisualization to reveal urban changes: Milan, Italy, 1737-2005. Cartographica: The International fournal for Geographic Information and Geovisualization 45, 1 (2010), 47-63. 

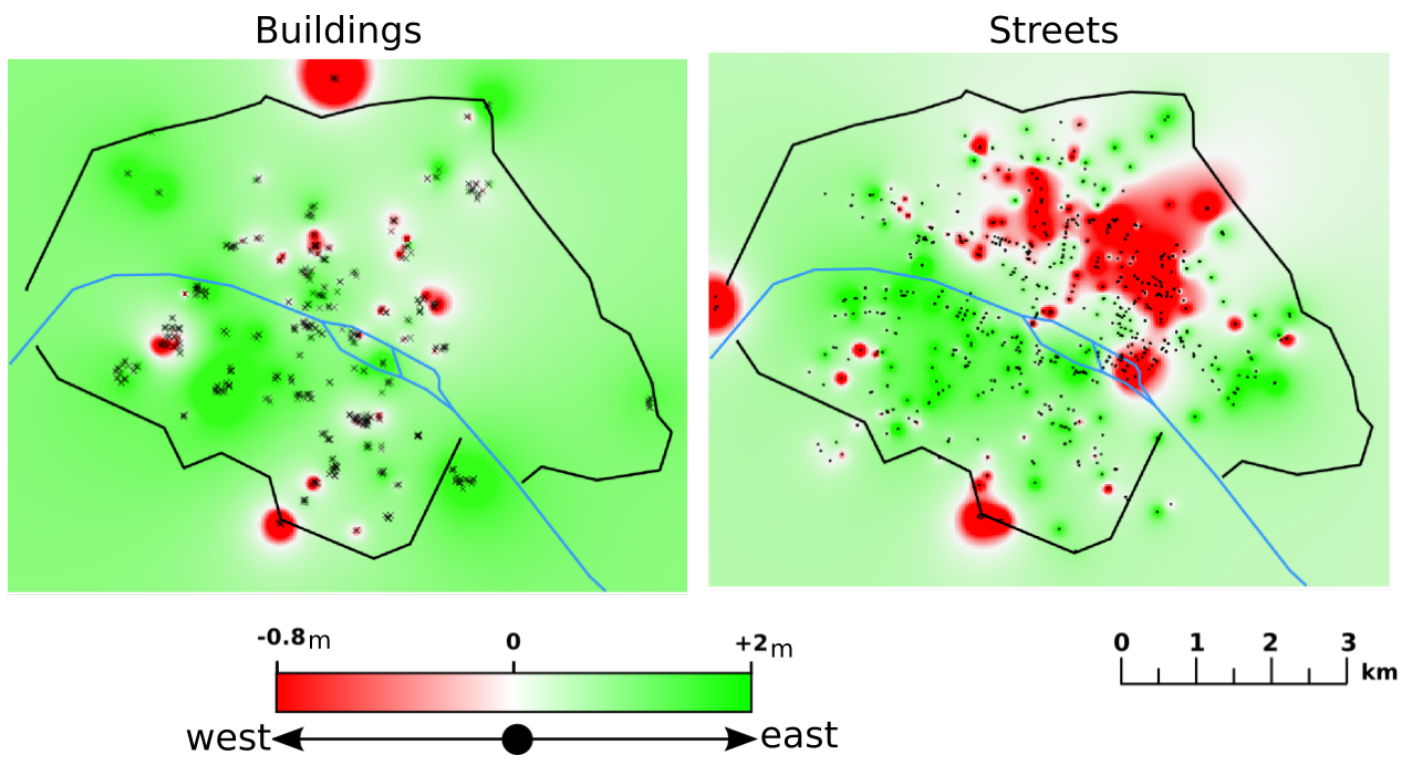

Figure 4: Mapping the horizontal component of the distortion vectors.

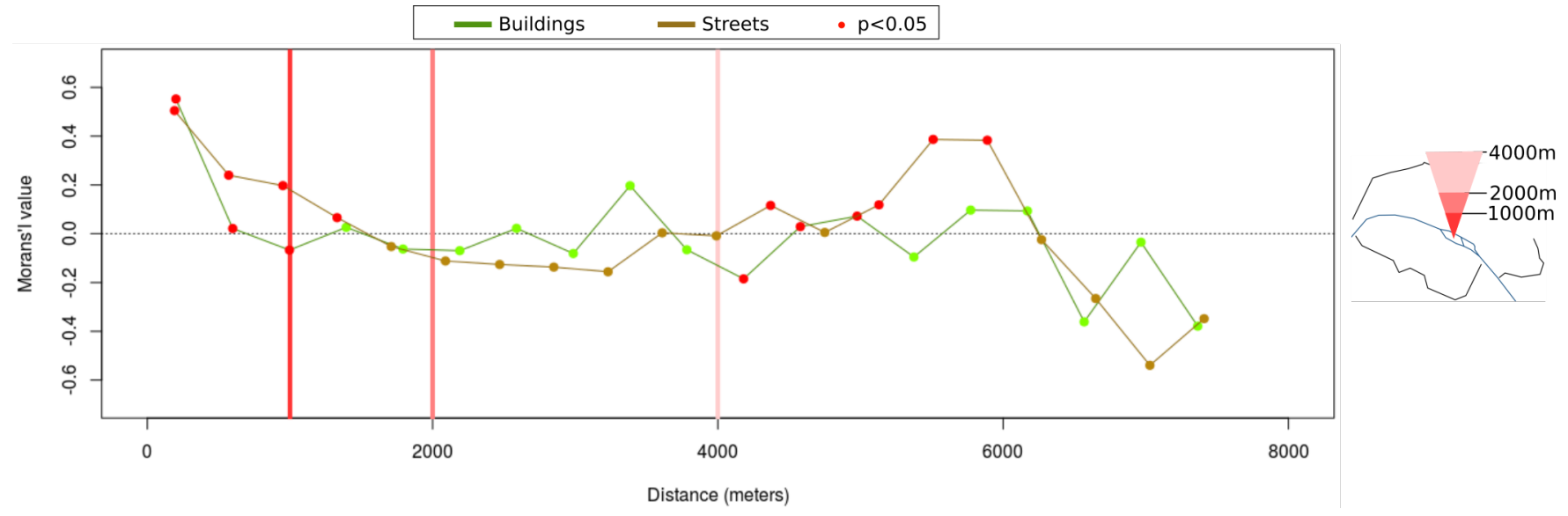

Figure 5: Correlogram of the horizontal component of the distortion vectors. 


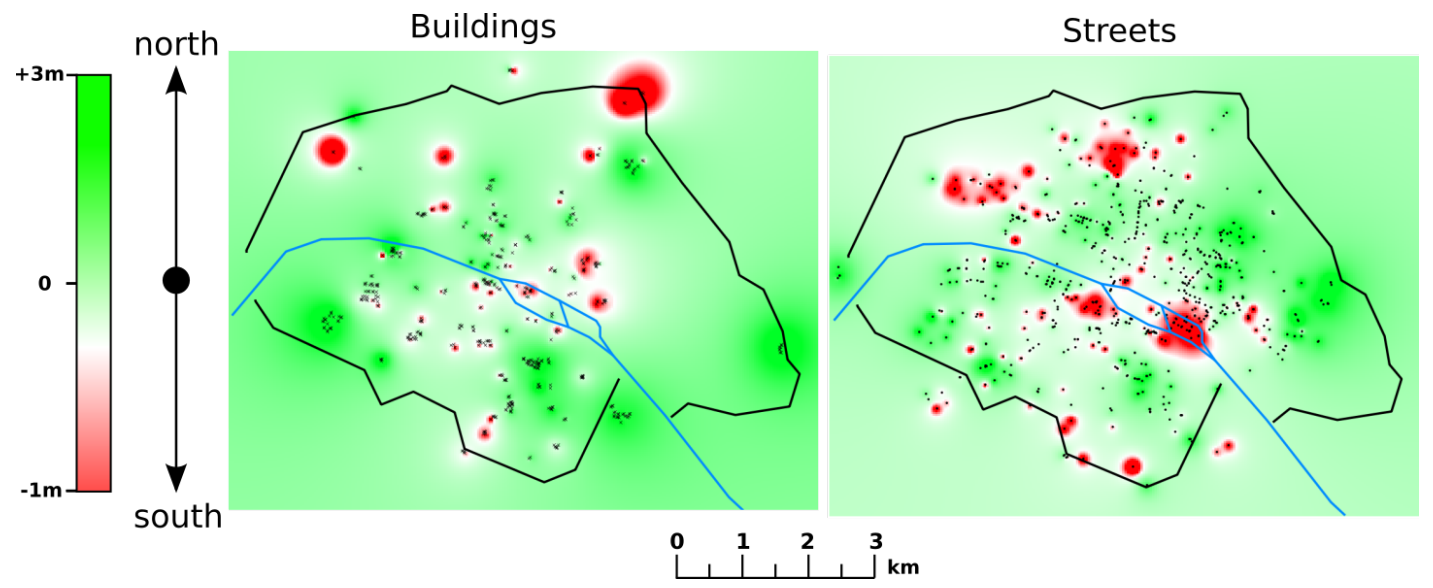

Figure 6: Mapping the vertical component of the distortion vectors.

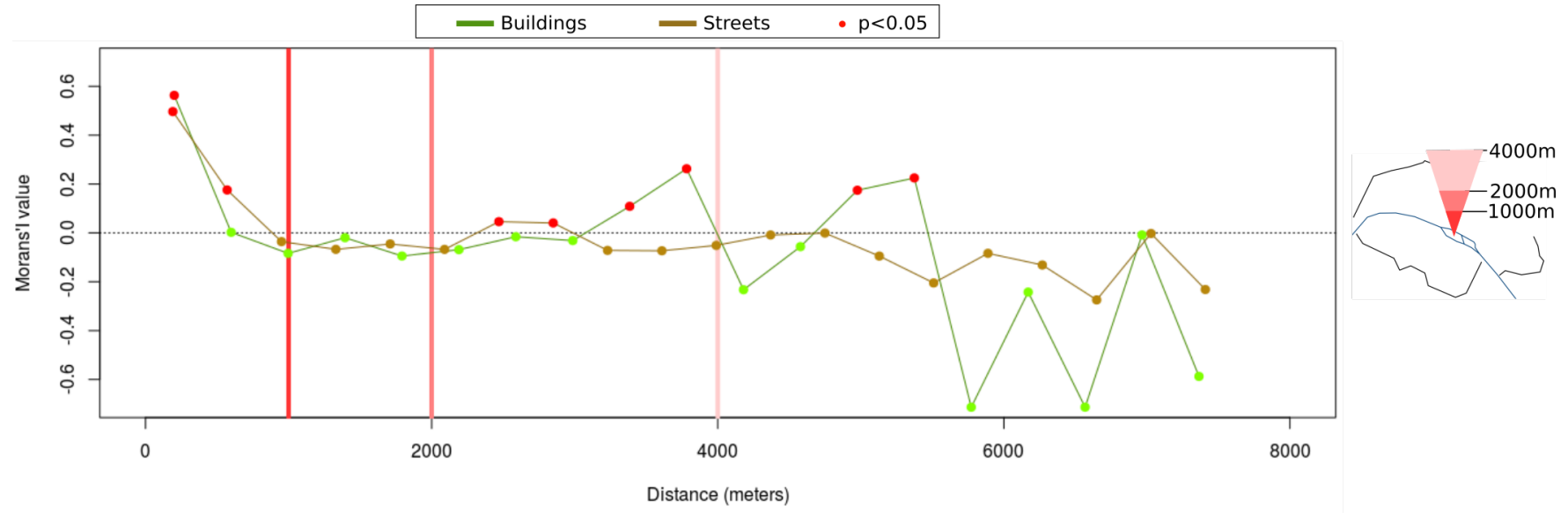

Figure 7: Correlogram of the vertical component of the distortion vectors. 